\title{
Original
}

\section{Effects of Local Administration of Novel Bisphosphonate Disodium Dihydrogen-4-[(Methylthio) Phenylthio] Methane- Bisphosphonate (MPMBP) on the Healing of Femoral Bone Defects in Wistar Rats}

\author{
Haruka Saito $^{1)}$, Yoshinaka Shimizu ${ }^{2}$, Takeshi Tone ${ }^{1)}$, Kenji Odashima ${ }^{1)}$, Yuya Sano ${ }^{2)}$, \\ Hisashi Shinoda ${ }^{3)}$, Tetsu Takahashi ${ }^{1)}$ and Hiroyuki Kumamoto ${ }^{2)}$ \\ 1) Division of Oral and Maxillofacial Surgery, Tohoku University Graduate School of Dentistry, Sendai, Japan \\ 2) Division of Oral Pathology, Tohoku University Graduate School of Dentistry, Sendai, Japan \\ ${ }^{3)}$ Center for Environmental Dentistry, Tohoku University Graduate School of Dentistry, Sendai, Japan \\ (Accepted for publication, March 16, 2019)
}

\begin{abstract}
The osteogenic effect of newly developed bisphosphonate (Disodium Dihydrogen-4-[(methylthio) phenylthio] Methane- Bisphosphonate (MPMBP)) was examined for clinical application in bone damage. A bone defect was made in the femur of ninety-seven male Wistar rats. $30 \mu 1$ of MPMBP (experimental group) or saline (control group) was injected around defect once every 3 days. The osteogenic effect to 4 weeks was performed at 1, 2, 3 and 4 weeks (experiment 1). Long-term observation was performed at 12 and 24 weeks after discontinuation of administration of MPMBP at 3 weeks (experiment 2). Newly formed bone was examined radiographically and histologically in both experiments. The elemental analysis was performed to investigate the quality of bone by energy-dispersive X-ray analysis. In experiment 1 , newly bone formation is observed in the bone marrow cavity and cortical defect in both groups. The bone volume at 4 weeks was decreased in control group and maintained in experimental groups. In experiment 2, newly formed bone in bone marrow cavity was absorbed at 12 weeks in control group, and showed gradually decrease to 24 weeks in experimental group. The $\mathrm{Ca} / \mathrm{P}$ of newly formed bone showed little difference between control and experimental groups. It was confirmed that the MPMBP administration had local effects, showing the promotion of new bone formation and long-term bone remodeling.
\end{abstract}

Key words: Disodium dihydrogen-4-[(methylthio) phenylthio] methane- bisphosphonate (MPMBP), Bisphosphonate, Bone remodeling, Bone formation.

\section{Introduction}

Clinically, bone defect healing is often required for trauma that has caused bony disjunction and surgical interventions that are intended to create bony injury with the aim of inducing a repair response or a therapy, such as treatment of tumor, cyst, infection, extraction of tooth, fixation of implant, fusion operation, maintenance of grafted bone and so forth ${ }^{1)}$. Various approaches ranging from cell-based to scaffold based strategies have been made for the therapy of such bone loss ${ }^{2}$. The basic idea of these strategies is to obtain positive calcium balance between bone resorption and bone formation at the local site, i.e., if more bone is formed than resorbed, an increase in the bone mass can be achieved.

Bisphosphonates (BPs) have been successfully used for the treatment of various metabolic bone diseases, such as osteoporosis, Paget's disease and cancer related distraction of bone, which are characterized by excessive bone $\operatorname{loss}^{3)}$. Due to their potent inhibitory effects on osteoclastic bone resorption, BPs are known to increase bone mass by changing the calcium balance from negative to positive, when administered systemically $^{4)}$. Since BPs have strong affinity for bone mineral and stay long where they were administered, they can act not only systemically,

Correspondence to: Dr. Haruka Saito, Division of Oral and Maxillofacial Surgery, Tohoku University Graduate School of Dentistry, 4-1 Seiryomachi, Aoba-ku, Sendai 980-8575, Japan; Phone: +81-22-717-8350; Fax: +81-22717-8359; E-mail: h.saitoh@dent.tohoku.ac.jp but also topically at the local site of administration. In fact, several reports have shown that topical BP can prevent alveolar bone loss in periodontitis $^{5)}$, augment fixation of implants ${ }^{6}$, and prevent orthodontic tooth movement ${ }^{7)}$ at the local site of administration.

BPs are chemically characterized by a P-C-P bond and this structure allows us to create a great number of variations by changing two lateral side chains on the carbon atom. Many BPs have been synthesized and investigated with respect to their effects on bone metabolism ${ }^{8}$. The data show that the pharmacological characteristics of bisphosphonates, such as effects on the balance between bone formation and resorption, mechanism of action, their potency, and side effects, vary depending on the structure of side chains ${ }^{9,10)}$.

[4-(Methylthio)phenylthio] methanebisphosphonate (MPMBP) is a novel bisphosphonate with an anti-oxidant side chain on the carbon atom of the P-C-P bond ${ }^{11)}$. Unfortunately it has not been published, during the course of our studies on the structure-activity relationships of various bisphosphonates, we recently found that this MPMBP has not only anti-bone-resorbing action, but also has potent anabolic action on bone. Such a compound could be effective for the treatment of extensive loss of osseous tissue which requires rapid gain of bone.

We hypothesized that topical administration of such MPMBP may induce the increase in the bone mass during healing process of bone defect. The current study was, thus, carried out to investigate effects of 
topical MPMBP on the relative gain/loss of newly-formed bone in a bone defect created in rat femur.

\section{Materials and Methods \\ Drug \\ [4-(Methylthio)phenylthio] methanebisphosphonate (MPMBP) was} used in the present study (Fig.1). This compound was synthesized in the Division of Organic and Bioorganic Chemistry, Department of Medicinal Chemistry, School of Pharmacy, Showa University (Tokyo, Japan) ${ }^{11)}$, and kindly supplied for us.

MPMBP solution at a concentration of $20 \mathrm{mM}$ was prepared by dissolving MPMBP in distilled water. The solution was adjusted $\mathrm{pH}$ to $7.2-$ 7.4 with $\mathrm{HCl}$ or $\mathrm{NaOH}$, and osmolality to 310 mOsm with $\mathrm{NaCl} .0 .9 \%$ $\mathrm{NaCl}$ solution was also prepared and served for control solution. All the solutions used were sterilized by filtration (Pore size $0.22 \mu \mathrm{m}$, SANPLATEC CORP., Osaka, Japan) and used for the topical injection in the following animal experiments.

\section{Animal experiments}

Ninety-seven male Wistar rats (Kumagai-shigeyasu Co.,Ltd, Sendai, Japan) ranging from 15 to 21 weeks of age, with an average body weight of $331 \mathrm{~g}$ were raised individually or by pairs in a cage. The rats were allowed to free access to food and water, and had the habituation period with 12/12 hour light-dark illumination cycle for one week or more. Under general anesthesia, (Isoflurane, Inhalation anesthesia, Inducation of anesthesia; 5\%, Maintenance of anesthesia; 1.5 2.0\%) a bone defect with a diameter of $1.5 \mathrm{~mm}$ and a depth of $3 \mathrm{~mm}$ was created in the diaphysis of both the right and left side femoral bone on the lateral side using a special bur for dental engine (a diameter of $1.45 \mathrm{~mm}$ and a length of $3.0 \mathrm{~mm}$ tungsten carbide bur).

After the operation, the above animals were divided into 2 groups and served for the following 2 experiments.

\section{Experiment 1: Effect of topical administration of MPMBP for 1-4 weeks on the bone defect}

Of the above 97 rats, 61 rats were served for this experiment. They were subdivided into experimental and control groups. Thirty-one rats in the experimental group were given sub-peritoneal topical injection of $30 \mu \mathrm{l}$ of $20 \mathrm{mM}$ MPMBP solution subjacent to the bone defect in the femur once every 3 days (under isoflurane inhalation anesthesia). The topical administration of MPMBP was started on the day when the bone defect was created. They were killed 1, 2, 3, or 4 weeks after the beginning of experiment.

Thirty rats in the control group were administered topically with 30 $\mu 1$ of $0.9 \% \mathrm{NaCl}$ solution in the same way as the administration of MBMBP solution and were killed on the same day as the rats in the experimental group (Fig.2).

Experiment 2: Long-term observation of the bone defect after the topical administration of MPMBP

Thirty-six rats were used in this experiment. They were subdivided into experimental and control groups. Eighteen rats in the experimental group were administered with $30 \mu 1$ of $20 \mathrm{mM}$ MPMBP solution once every 3 days for 3 weeks as in Experiment 1. Eighteen rats in the control group were administered with $30 \mu \mathrm{l}$ of $0.9 \% \mathrm{NaCl}$ instead of MPMBP. After the administration of MPMBP or $0.9 \% \mathrm{NaCl}$ for 3 weeks, all the rats were maintained as they were until the end of experiment. The rats in both experimental and control groups were killed at 4, 12 or 24 weeks after the beginning of experiment (Fig. 2).

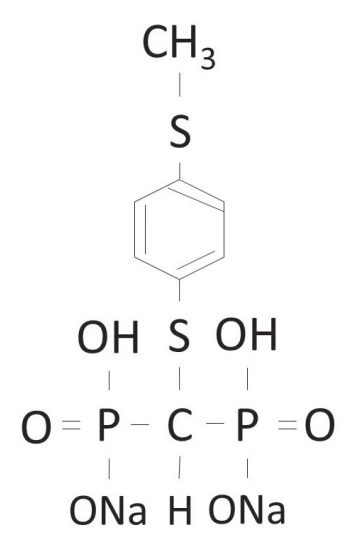

A; MPMBP

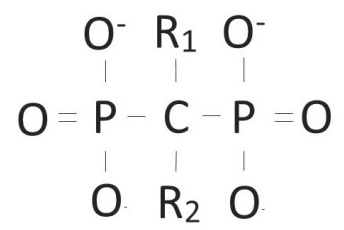

B; bisphosphonate
Figure 1. Chemical structure of MPMBP

In the present animal experiments, an excessive pentobarbital was used when the rats were killed. Fixation of femoral bone was performed by perfusing $4 \%$ paraformaldehyde (PFA) from the heart. All femurs with bone defect were dissected and soaked in 4\% PFA and subjected to the following micro-computed tomography, histological and histochemical examinations, and energy-dispersive X-ray analysis.

All the animals in the present study were treated ethically according to the guidelines of the Institute for Animal Experimentation of Tohoku University Graduate School of Medicine. The study protocol and experimental design were reviewed and approved (2015DnA-032) by The Animal Research Committee of Tohoku University.

\section{Micro-computed tomography (micro-CT)}

Micro-computed tomographic (CT) images of the bone defects were obtained using a micro-CT scanner (voltage: $90 \mathrm{kV}$, current: $100 \mu \mathrm{A}$, pixel size: $18 \mu \mathrm{m} /$ pixel) (ScanXmate-E090, Comscantechno Co., Ltd.). Transparent images of whole femoral bones were also taken by the device. The total length of the femoral bone and breadth of femoral diaphysis were measured using the transparent image (Fig. 3). The volume of newly formed bone in the part of cortical bone defect and inside the bone marrow cavity were measured based on the $\mathrm{CT}$ image using an ImageJ software (National Institutes of Health, Bethesda, Maryland, U.S.); the radiopaque area of newly formed bone in the marrow cavity and cortical defect area were measured. The volume percentage of newly formed bone against initial bone marrow cavity was also calculated. Three-dimensional image of the area of bone defect was created using VG Studio Max (Volume Graphics, Heidelberg, Germany) software package.

\section{Histological and histochemical examinations}

Femoral bones dissected were fixed in 4\% PFA, decalcified with $10 \%$ ethylenediamine-tetraacetate (EDTA), and embedded in paraffin. Longitudinal histological sections of bone defect with a thickness of 4 $\mu \mathrm{m}$ were prepared. They were stained with hematoxyline and eosin (H\&E), with the method of Elastica-Masson (EM) staining, alkaline phosphatase (ALP) staining, and tartrate-resistant acid phosphatase (TRAP) staining.

\section{Elastica-Masson (EM) staining}

Paraffin-embedded serial sections were deparaffinized with xylene and hydrated with an ethanol gradient (100\%-70\%). Slides were oxi- 


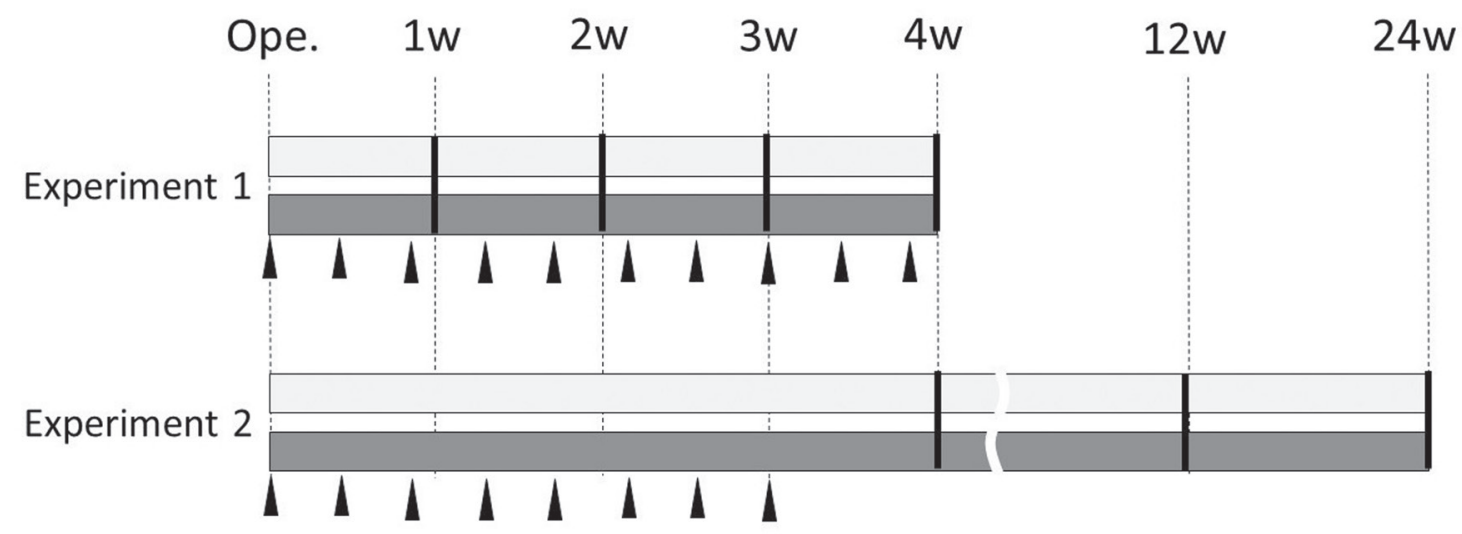

Experimental group $\square$ Control group

Application saline or MPMBP $\quad$ | Sacrifice
30ul/3days

Figure 2. Experiment schedule

dized with dichromic acid for 12 hours and were rinsed in ion-exchanged water. They were stained with resorcin-fuchsin for 1 hour followed by 15 dips in $1 \%$ acid ethanol $(1 \% \mathrm{HCl}$ in $70 \%$ ethanol) and then rinsed in ion-exchanged water. They were stained with hematoxylin for $5 \mathrm{~min}$ followed by 15 dips in $1 \%$ acid ethanol and then rinsed in ion-exchanged water. After staining with ponceau-fuchsin for $30 \mathrm{~min}$ and rinse in acetic acid water, similarly, they were stained with orange- $G$ for 15 min and light-green for $5 \mathrm{~min}$, and rinsed in acetic acid water each time.

\section{Alkaline phosphatase staining}

After deparaffinization by immersion in xylene and ethanol of serial sections, slides were rinsed in distilled water and incubated with activation solution (50 mM magnesium chloride solution) overnight at $4{ }^{\circ} \mathrm{C}$. Then, they were rinsed with $0.1 \mathrm{M}$ Tris- $\mathrm{HCl}$ buffer $(\mathrm{pH} 8.5)$ and incubated with ALP reaction solution, that were mixed naphtol AS-BI phosphate (Sigma-Aldrich Co. LLC., Darmstadt, Germany), N, N-dimethylformamide (Sigma-Aldrich Co. LLC., Darmstadt, Germany), 0.1M tris$\mathrm{HCl}$ buffer and fast blue BB salt (Sigma-Aldrich Co. LLC., Darmstadt, Germany), for $30 \mathrm{~min}$ at $37^{\circ} \mathrm{C}$.

\section{Tartrate-resistant acid phosphatase (TRAP) staining}

After deparaffinization by immersion in xylene and ethanol of serial sections, slides were rinsed in $0.1 \mathrm{M}$ acetate buffer ( $\mathrm{pH}$ 5) and incubated with reaction solution, that were mixed $50 \mathrm{mM}$ tartaric acid, magnesium chloride hexahydrate, N, N-dimethylformamid (Sigma-Aldrich Co.

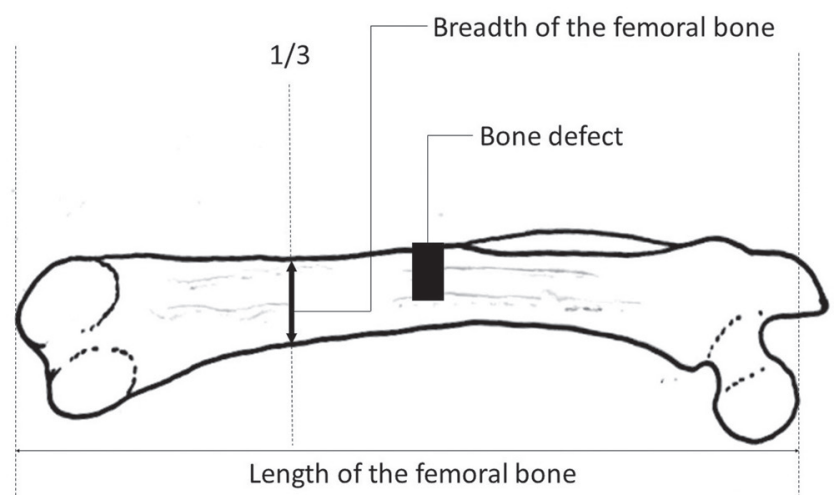

Figure 3. Length and breadth of the femoral bone
LLC., Darmstadt, Germany), naphthol AS-MX phosphate (Sigma-Aldrich Co. LLC., Darmstadt, Germany), fast red violet LB salt (Sigma-Aldrich Co. LLC., Darmstadt, Germany), for $30 \mathrm{~min}$ at $37^{\circ} \mathrm{C}$. After rinse in distilled water, they were counterstained with hematoxylin for $15 \mathrm{~min}$ and rinsed in distilled water, soaked in distilled water for $30 \mathrm{~min}$.

The volume and the surface length of newly formed bone in the bone defect were measured (KURABO, Osaka, Japan). Activity of osteoblasts on the newly formed bone was histochemically examined using

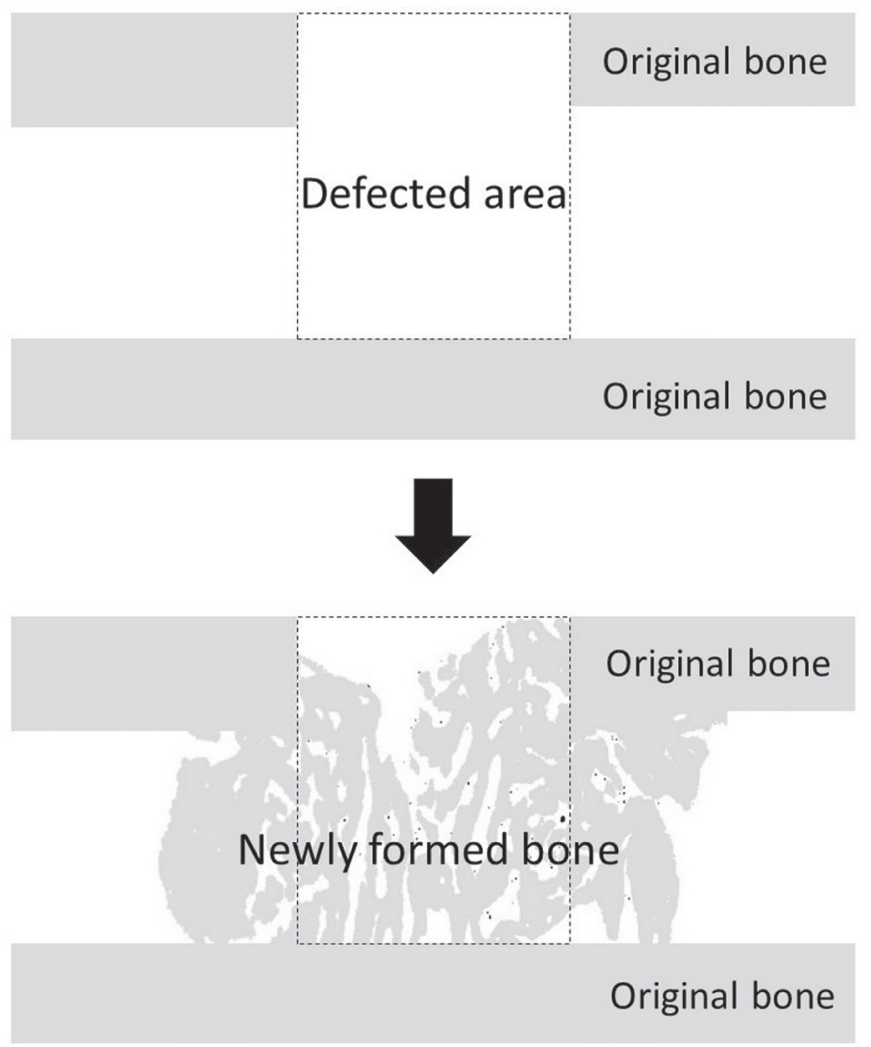

Figure 4. Scanning area of energy-dispersive X-ray analysis (EDX) 


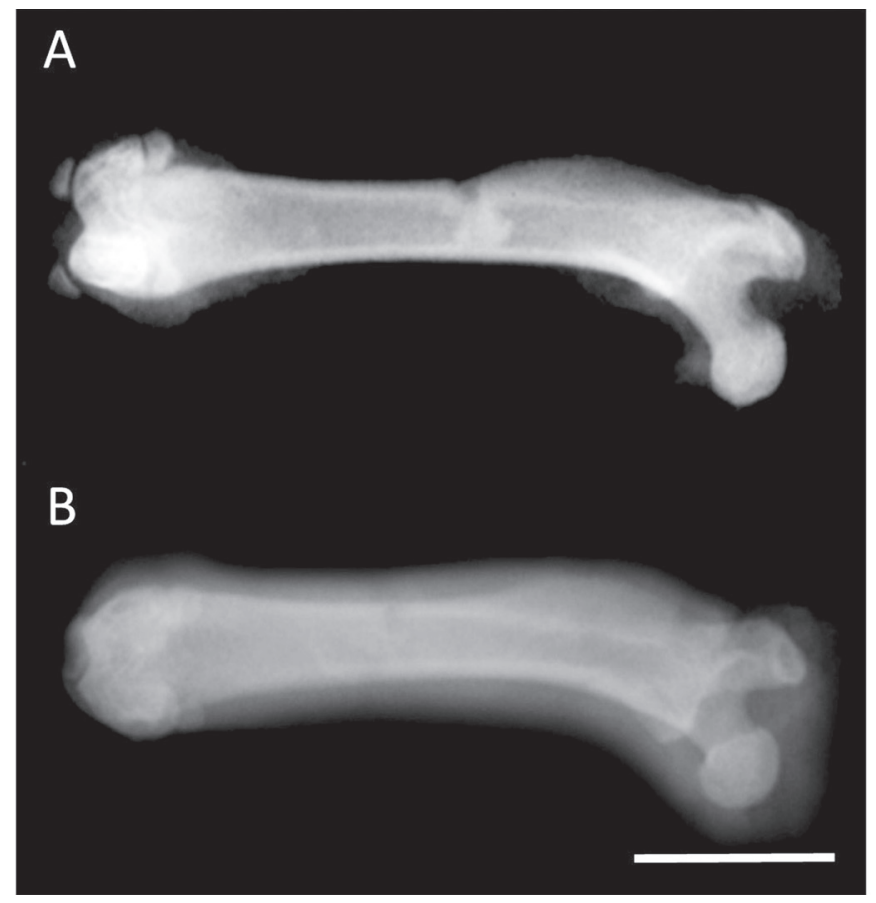

Figure 5. Radiographical image. A; control group at week 3, B; experimental group at week 3 . Bar $=10 \mathrm{~mm}$

staining images of alkaline phosphatase activity (ALP). The number of osteoclasts per unit surface of newly formed bone in the bone defect was also measured using staining image of the activity of tartrate-resistant acid phosphatase (TRAP).

\section{Energy-dispersive X-ray analysis (EDX) of newly formed bone}

Four samples from experiment1 (topical administration 2 weeks and 4 weeks in each group (experimental and control)) were fixed by $10 \%$ formalin solution, dehydrated in a graded ethanol series, and embedded in methyl methacrylate. They were cut using Leica SP1600 Saw Microtome and coated with carbon. Samples were examined by a scanning electron microscope coupled with energy-dispersive X-ray analysis (EDX) (JSM-6300LA, EX-2300, JEOL, Tokyo, Japan), with $20 \mathrm{~mm}$ working distance, $15 \mathrm{kV}$ acceleration voltage, and $\times 15$ magnification. To examine the quality of bone, the elemental composition $(\mathrm{Ca} / \mathrm{P}$ ratio) of the newly formed bone in the bone defect was measured and com- $[\mathrm{mm}]$

A

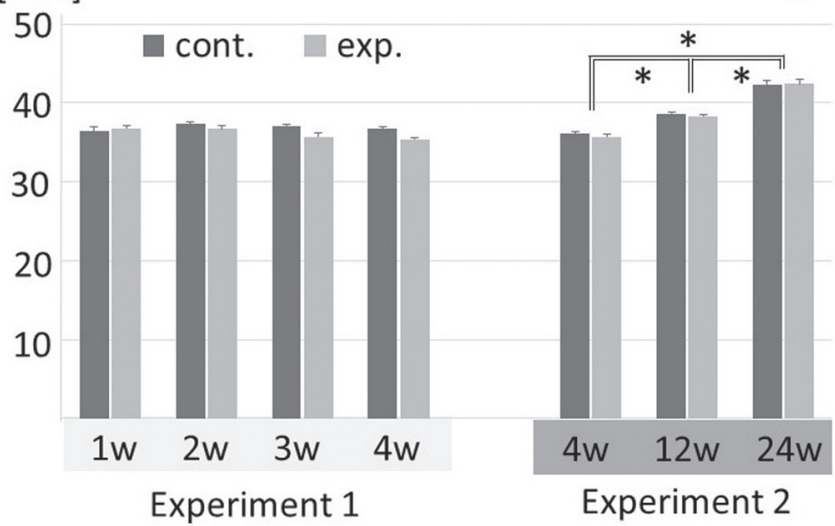

$[\mathrm{mm}]$

B

50

40

cont. $\quad$ exp.
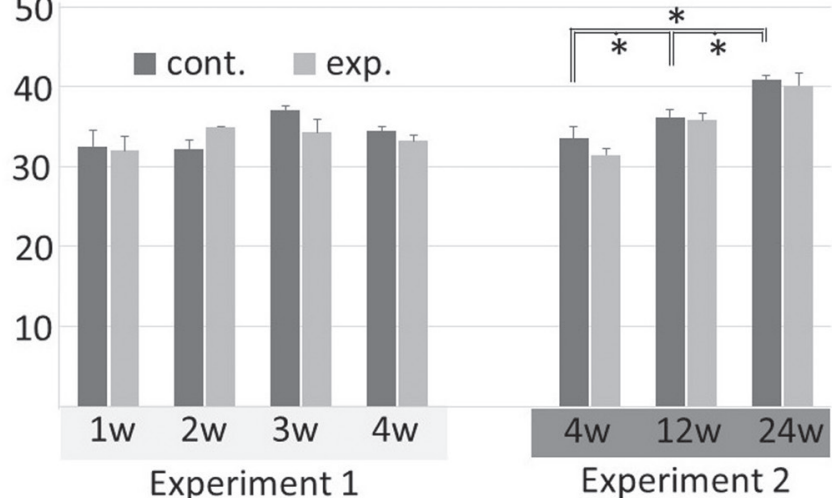

Experiment 2

Figure 6. Length and breadth of the femoral bone. A: Length, B: Breadth, *; $\mathrm{p}<0.05$

pared with that of original cortical bone (Fig. 4).

\section{Statistical analysis}

The data obtained by micro-computed tomographic analysis and histological examinations were subjected to two-way analysis of valiance (2-way ANOVA), followed by Scheffe's multi-comparison test. $\mathrm{p}<0.05$ was considered a significant difference.

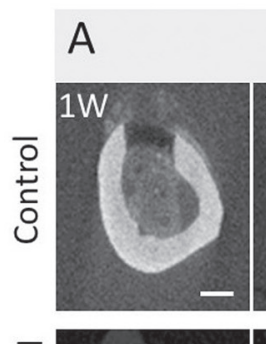

\section{Experiment 1}
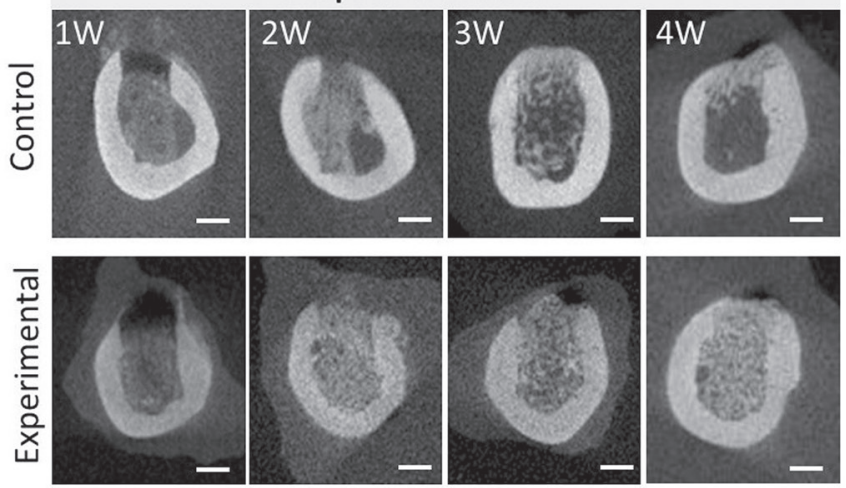

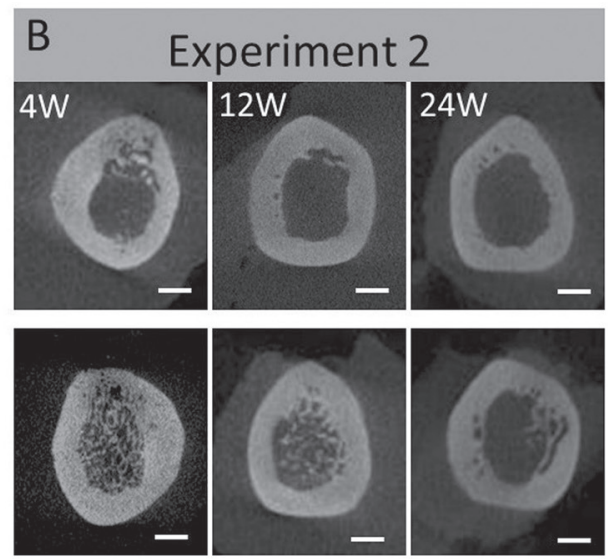

Figure 7. Micro-CT image. A: experiment 1, B: experiment 2. Bar=1 mm 


\section{Results}

\section{Effects of MPMBP on the length and breadth of the femoral bone}

The fluoroscopic X-ray images are shown in Fig. 5. All of the X-ray fluoroscopic images of the femur showed no abnormal structure or growth with pathological change (Fig. 5).

Time-dependent changes in the length and breadth of the femoral bone are shown in Fig. 6.

In the experiment 1 , the average length and breadth of the femoral bones showed no significant increase ranging from week 1 to 4 , with no significant difference between the control and experimental groups (Fig. $6 \mathrm{~A}$ and $\mathrm{B})$.

In the experiment 2 , the average of both length and breadth of the femoral bones significantly increased ranging from week 4 to 24 $(p<0.05)$, with no significant difference between the experimental group and control group.

Effects of MPMBP in the experiment 1, topical administration for 1-4 weeks.

Micro-CT images

Fig. 7 shows the horizontal sectional images using micro-CT in the experiment 1. At week 1, radiopaque areas due to newly formed bone were seen in the bone marrow cavities and cortical defect areas in both groups. In the experimental group, the radiopaque area in the bone marrow cavity increased over week 2 , maintaining until week 4 . The radiopaque area in the cortical bone defect showed the same change as that in the bone marrow cavity. In the control group, the radiopaque area in the bone marrow cavity increased over week 2 and decreased at week 3 and 4. The radiopaque area in the cortical bone defect showed apparent increases at week 2 and 3 and maintained unchanged at week 4 .

\section{Three dimensional image of the area of the bone defect and injection site}

Three dimensional images of the area of the cortical bone defect near the injection site are shown in Fig. 8. In the control and experimental groups, the parts of cortical bone defects were observed with the concave bone surfaces at week 1. However, in the experimental group, external bone formation was observed nearby the injection site at week 4.

\section{The volume of newly formed bone using micro-CT image}

The volume percentage of newly formed bone in the bone marrow cavity and cortical bone defect is shown in Fig. 9 and Fig. 10. In the experimental group, the volume percentage of newly formed bone in the bone marrow cavity was approximately $60 \%$ until week 3 and showed a slight decrease over week 4 . In the control group, the volume percentage of newly formed bone in the bone marrow cavity was $60 \%$ until week 2 and showed apparent decreases at week 3 and $4(p<0.05)$. At week 4 , significant differences were seen between the experimental and control groups ( $\mathrm{p}<0.05$ ).

The volume of newly formed cortical bone markedly increased with time in the control and experimental groups $(\mathrm{p}<0.05)$.

\section{Histological examinations}

Fig. 11 and Fig. 12 show histological images. In the experimental group, at week 1, newly formed bone was seen in the bone marrow cavity at week 1 and hardly absorbed, lasting in the bone marrow cavity at week 4. On the other hand, in the control group, newly formed bone was mostly absorbed by week 4 . No abnormal inflammatory cells infiltration around the injection site was observed in both groups.

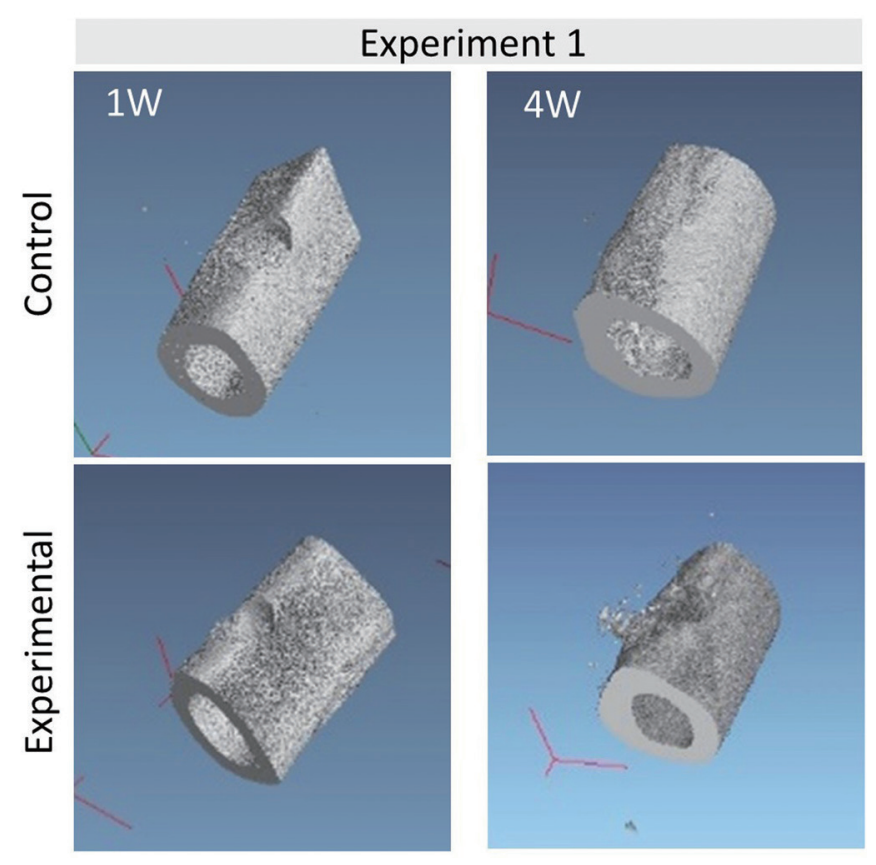

Figure 8. Three dimension structure
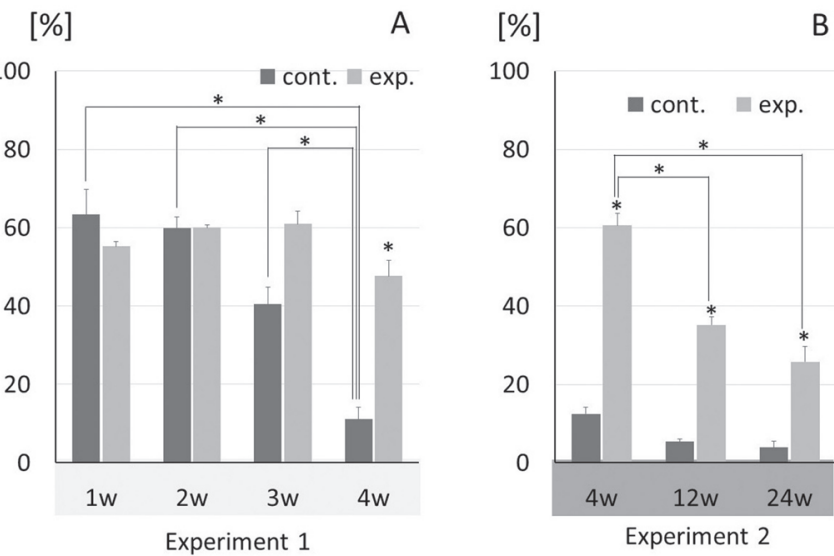

Figure 9. Newly formed bone volume in the bone marrow cavity. A: experiment 1 , B: experiment 2. *; $\mathrm{p}<0.05$
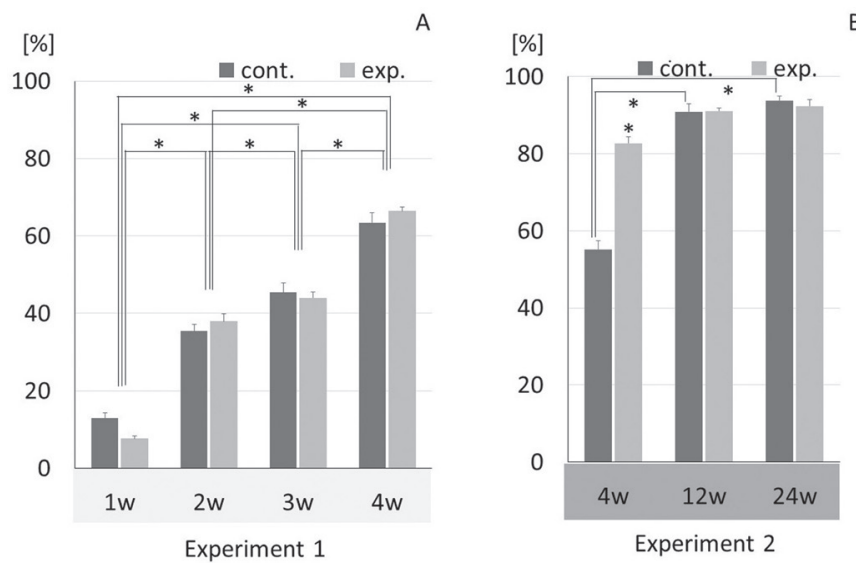

Figure 10. Newly formed bone volume in the cortical bone defect. A: experiment 1 , B: experiment $2 * ; \mathrm{p}<0.05$ 


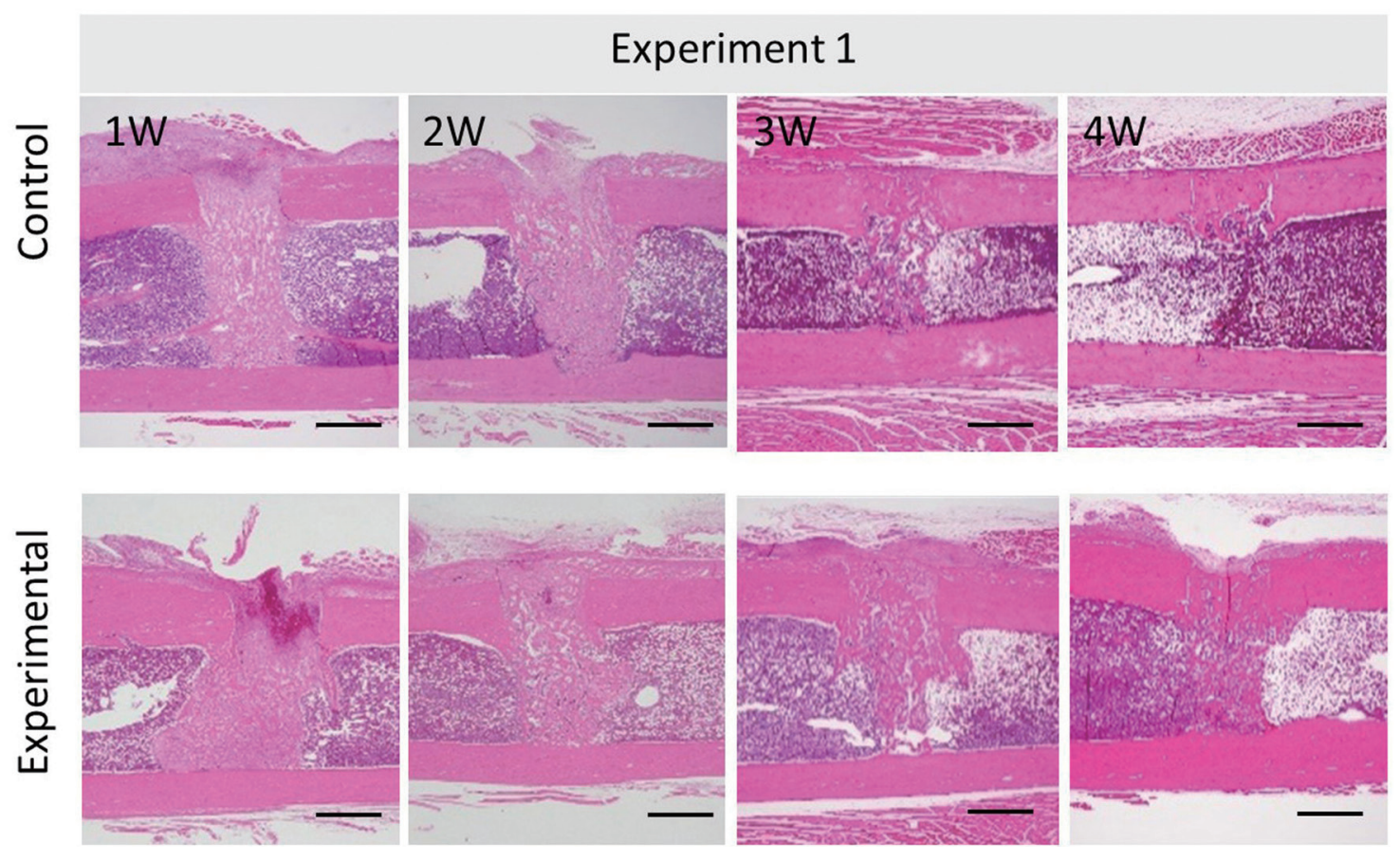

Figure 11. H\&E staining images in experiment 1 , bar=1 mm.

EM staining images revealed that newly formed bone was irregular netlike trabecular without lamellar structure and rich in collagen fibers.

ALP staining images revealed that the surfaces of newly formed bone in the control and experimental groups were stained with a blue color, which indicated alkaline phosphatase activity (Fig. 12). At week 4, ALP activity in the remaining new bone apparently decreased in both groups compared to that at week 1. TRAP-positive osteoclasts, which was stained red, were diffusely observed on the new bone surface (Fig. 12). The number of the osteoclasts observed at week 4 was fewer than that at week 1 . The number of osteoclasts in the experimental group was fewer than that in the control group.

\section{Histomorphometrical examinations}

Fig. 13A shows the bone volume of newly formed bone in the bone marrow cavity. In the experimental group, increased bone volume was observed at week 3 and 4 with no significant difference while in the control group, the bone volume significantly decreased ranging from week 1 to $3(p<0.05)$. The bone volume at week 4 showed significant difference between the experimental and control groups $(\mathrm{p}<0.05)$.

The densities of TRAP-positive osteoclasts are shown in Fig. 14. The densities of the osteoclasts showed the peak at week 2 and 3 in the control and experimental groups, respectively. At week 2, the density of the control group showed significantly higher compared to that of the experimental group $(\mathrm{p}<0.05)$.

\section{Energy-dispersive X-ray analysis (EDX) of newly formed bone}

The elemental analysis showed a lower $\mathrm{Ca} / \mathrm{P}$ ratio in newly formed bone at week 2 compared to existing bone at week 2 . There was no difference in the experiment and control groups (Table. 1).

\section{Effects of MPMBP in the experiment 2, long-term observation Micro-CT findings}

Fig. 7B shows micro-CT images in the long term experiment. In the control group, radiopaque area in the bone marrow cavity hardly re-

\begin{tabular}{lcccc}
\multicolumn{5}{c}{ Table 1. Ca/P ratio } \\
\hline \multirow{2}{*}{ week } & $\begin{array}{c}\text { original } \\
\text { bone }\end{array}$ & $\begin{array}{c}\text { newly } \\
\text { formed } \\
\text { bone }\end{array}$ & $\begin{array}{c}\text { original } \\
\text { bone }\end{array}$ & $\begin{array}{c}\text { newly } \\
\text { formed } \\
\text { bone }\end{array}$ \\
\hline control & 1.39 & 1.23 & 1.46 & 1.46 \\
experiment & 1.40 & 1.29 & 1.42 & 1.42 \\
\hline
\end{tabular}

mained at week 4 , with little new bone at week 12. In the part of cortical bone defect, porous structure appeared newly formed bone at week 4, decreasing gradually at week 12 and 24 . In the experimental group, radiopaque area in the bone marrow cavity decreased gradually over time until week 24. At week 12 and 24, newly formed bone apparently remained, compared to the control groups. In the part of cortical bone defect, newly formed cortical bone was porous at week 4 , and the boundary between newly formed bone and existing cortical bone was unclear at week 12 and 24 .

\section{Three dimensional images of bone defect area and injection site}

It was impossible to distinguish newly formed bone in the part of the bone defect from the normal cortical bone because the structures of the both bones were similar to each other. No abnormal bone formation was observed in the injection site.

\section{Morphometry on CT-images}

The volume of newly formed bone in the marrow cavity and cortical bone defect is shown in Fig. 9 and Fig. 10, respectively. In the experimental group, the bone marrow cavity contained $10 \%$ of radiopaque area at week 1 and showed a high percentage of radiopaque area at week 4; however, the percentage decreased over time until week 24 . The percentage of radiopaque area in the bone marrow cavity was significantly higher than that in the control groups $(\mathrm{p}<0.05)$.

In the control and experimental groups, the percentage of newly formed bone volume in the cortical bone defect accounted for $90 \%$ at week 12 . At week 24, newly formed bone mostly occupied the defect 

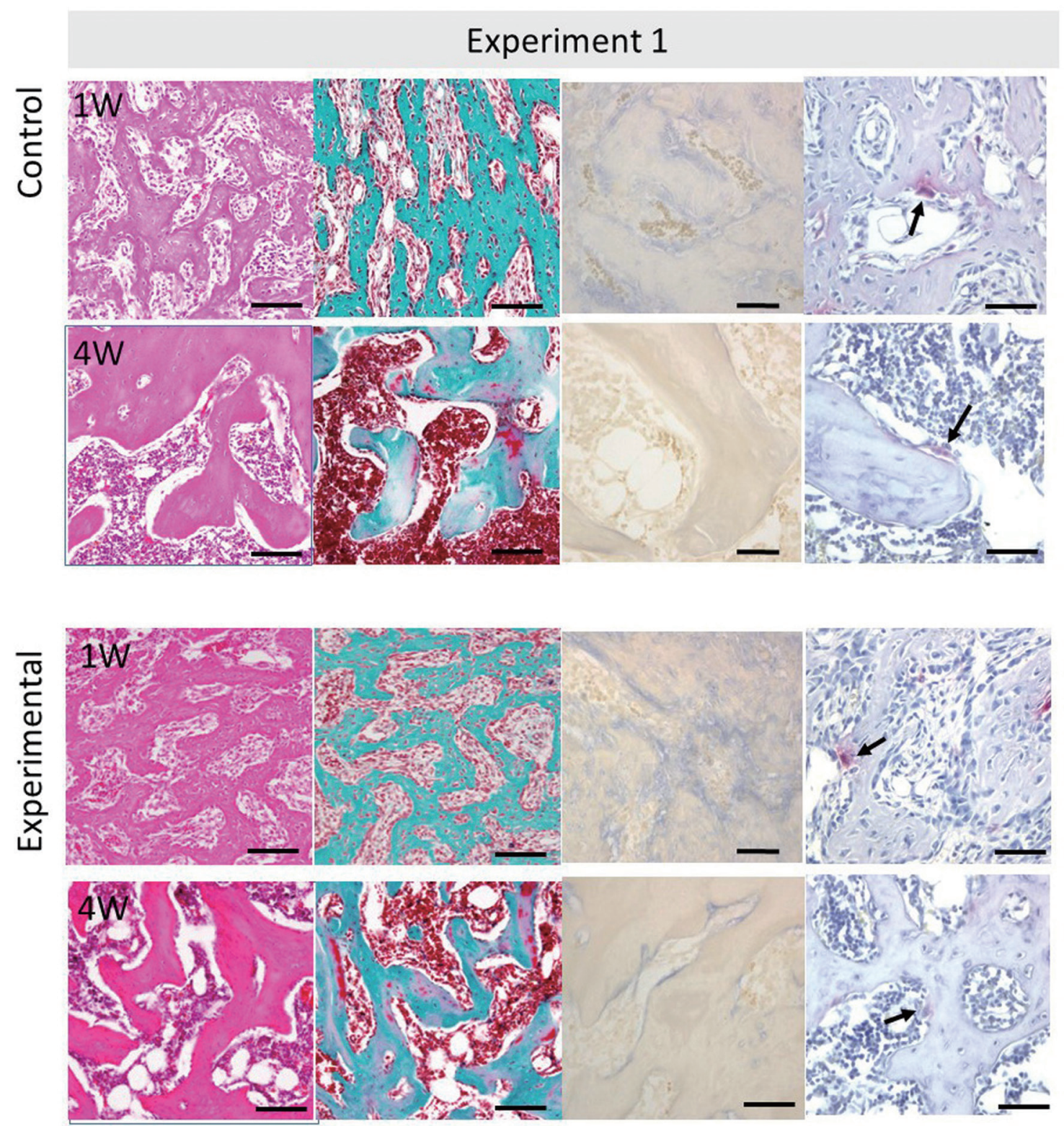

Figure 12. H\&E, EM, ALP, TRAP staining images at week 1 and 4 in the experiment 1 . H\&E, EM; bar=100 $\mu \mathrm{m}, \mathrm{ALP}, \mathrm{TRAP}$; bar $=50 \mu \mathrm{m}$. Arrow indicates TRAP positive osteoclast.

area. At week 4, the percentage of newly formed bone volume in the experimental group was higher than that in the control group (Fig. 10B).

\section{Histological examinations}

H\&E, ALP, TRAP staining images are shown in Fig. 11. In the experimental group, newly formed bone was seen in the bone marrow cavity at week 4 ; however, decreased newly formed bone volume was seen at week 12 and 24. In the control group, newly formed bone in the bone marrow cavity hardly remained at week 4 and was replaced with the bone marrow at week 12. In the control and experimental groups, newly formed bone in the cortical bone defect was porous at week 4 , and the boundary between the newly formed bone and cortical bone was unclear at week 12 and 24. ALP staining images demonstrated that the surface of remaining new bone had ALP activity, which was slightly higher in the experimental group compared to that of the control group.

TRAP staining images demonstrated that TRAP-positive osteoclasts appeared diffusely on the surface of remaining new bone. In the experimental group, more osteoclasts were observed on the surface of the remaining bone compared to the control group.

\section{Histomorphometrical examinations}

The bone volume of newly formed bone in the bone marrow cavity is shown in Fig. 14B. In the experimental and control groups, the bone volume showed significant decreases at week 12 and $24(\mathrm{p}<0.05)$. The bone volume in the experimental group was significant larger than that in the control group at week 4, 12 and $24(\mathrm{p}<0.05)$.

Fig. 15 shows the density of TRAP-positive osteoclasts. The density of osteoclasts in the control and experimental groups significantly decreased at week 4, 12 and $24(\mathrm{p}<0.05)$. There was no significant difference in the density of the osteoclasts between the control and experimental groups. 

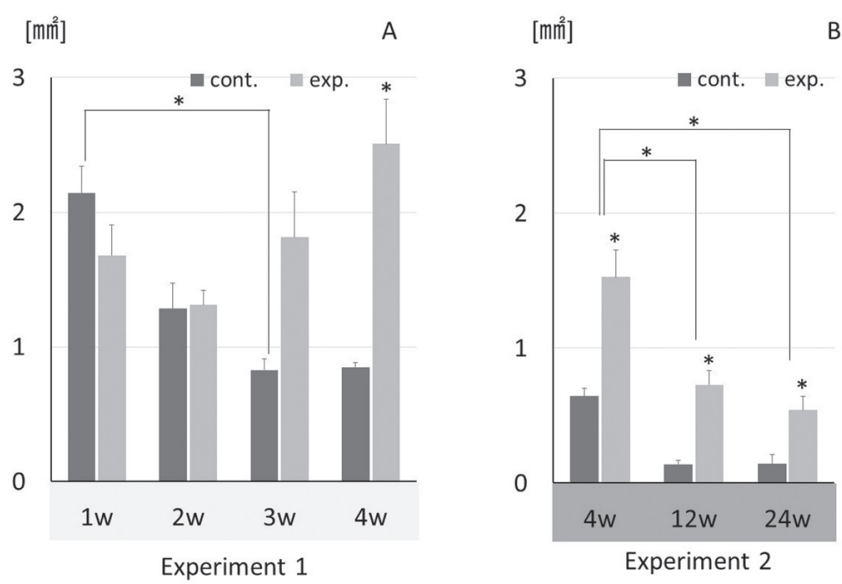

Figure 13. H\&E staining images of newly formed bone volume. A: experiment 1, B: experiment 2. *; $<<0.05$

$[/ \mathrm{mm}]$

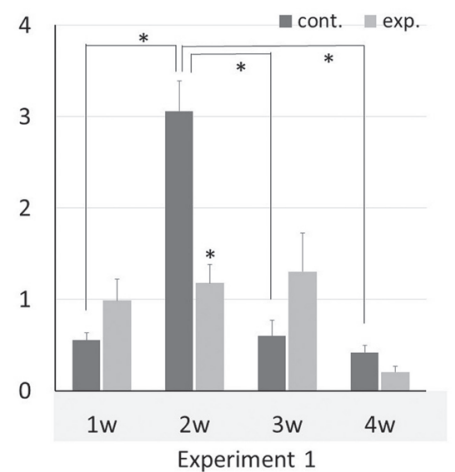
of MPMBP on the healing of femoral bone defects in rats.

All the tissue specimens revealed no remarkable inflammatory cell infiltration surrounding the bone defect part or abnormal absorption of the existing bone. It can be considered that MPMBP has no harmful effect that causes inflammation. The intravenous administration of zoledronate is reported to cause adverse reactions such as fever, atypical fracture of the femoral bone, osteomyelitis of jaw, and rarely seen, osteomyelitis of cranial bone ${ }^{12)}$. However, in this study, none of the following adverse reactions were observed: abnormal behavior with fever, atypical fracture of femoral bone or bone exposure with osteomyelitis. BRONJ is occurred by long term and ongoing administration of BP, that make harder bone without bone remodeling. However, in this study, short term administration of MPMBP showed bone resorption or remodeling in long-term observation. Therefore, our findings suggest that MPMBP administrated in short term will not cause BRONJ.

MPMBP can be considered to inhibit the absorption of new bone because in the short term experiment, no decreased volume of new bone in the bone marrow cavity was histologically observed since week 3 , being significantly different from the control group. Takizawa, et al. reported that whole body administration of MPMBP hindered bone resorption in OVX rats ${ }^{11)}$. Additionally, it was reported that in vitro and in vivo examinations showed the suppression of osteoclasts activity ${ }^{13,14)}$. Our results showed that the MPMBP suppressed the appearance and differentiation of osteoclasts in the administration site of the MPMBP. This meant that like other BPs, MPMBP inhibited not only whole body but local bone resorption. Shinoda et al. reported that the local administration of MPMBP (TRK-530) inhibited alveolar bone resorption in rats with periodontitis $^{15)}$. Combining the present results, MPMBP can have the effect of bone resorption inhibition.

Our histological results showed that the local administration of MPMBP had increased the new bone volume until week 4. Specifically, active bone formation inside the bone marrow cavity had been apparent since week 3 , which was the significant difference from the control group. According to Takizawa et al., MPBMP helped type 1 collagen to increasingly accumulate under the epiphyseal plate and also improved the bone volume and bone density in post-menopausal osteoporosis,

Figure 14. The density of TRAP/positive osteoclasts, A: experiment 1, B: experiment $2, * ; \mathrm{p}<0.05$

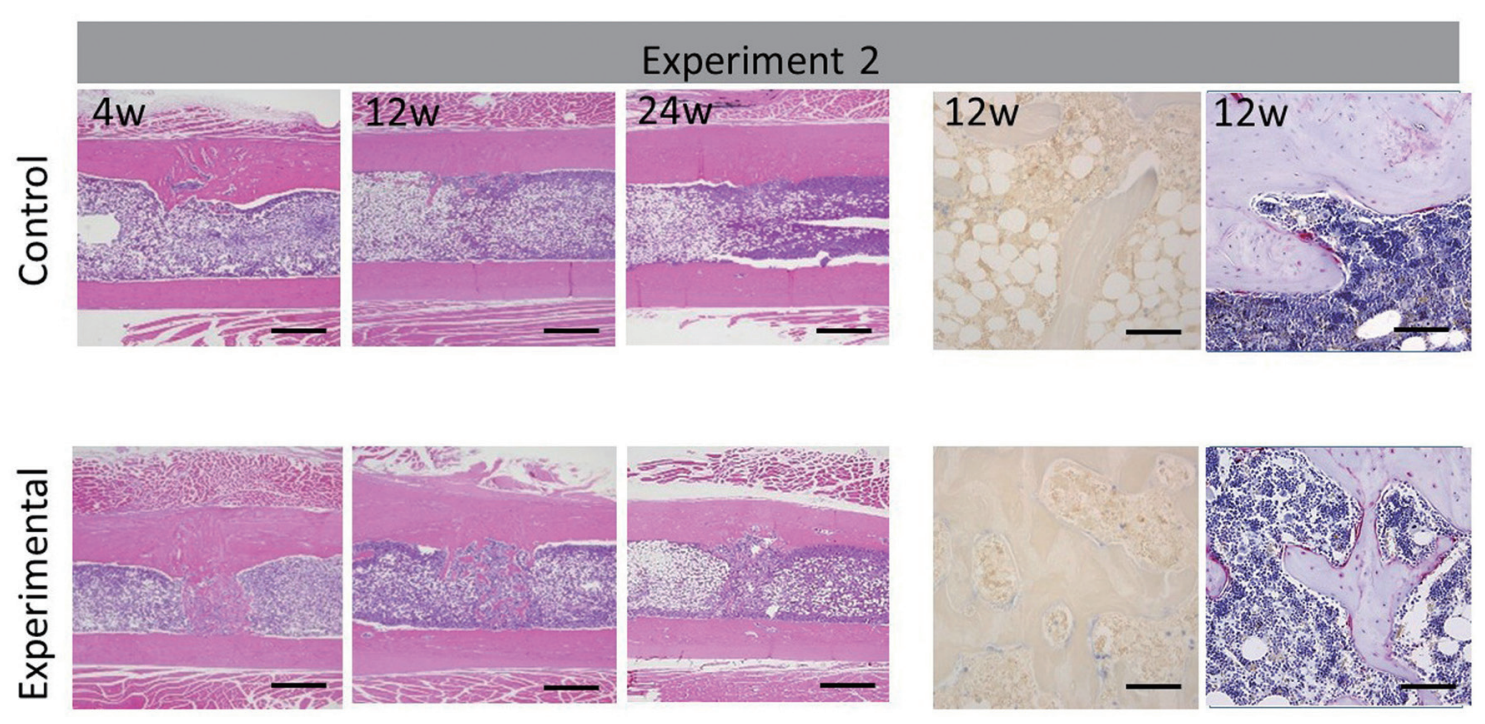

Figure 15. H\&E, ALP, TRAP staining images of the densities of in experiment 2. Bar=1 mm (H\&E), and $100 \mu \mathrm{m}(\mathrm{ALP}, \mathrm{TRAP})$. 
which was related to accelerating bone formation ${ }^{11)}$. Our findings support these previous reports and would demonstrate the favorable effect of MPMBP in other kinds of osteogenesis.

In the experiment 1 , micro-CT analysis indicated that the ratio of newly formed bone volume in the bone marrow cavity had no significant difference in week 1 and 2 while histological examination demonstrated the significant increase of newly formed bone volume. This may indicate that new bone is formed developmentally from woven bone, which is less calcified. Initial regenerated bone was composed of woven bone, which was replaced to the lamellar mature bone by bone remodeling ${ }^{16)}$. Newly formed bone in the initial stage of bone formation was shown to be immature bone by the elemental analysis. Therefore, it is considered that micro-CT image cannot identify the degree of mineralization of the bone.

It is necessary to assess the quality of the bone formed with MPMBP. If immature bone still remains instead of being replaced, the bone would lack hardness, which can cause pathologic fracture. In this study, EDX analysis revealed $\mathrm{Ca} / \mathrm{P}$ ratio had no difference between new bones with and without MPMBP administration in the short and long term experiments. Okata et al. reported that the bone was immaturely calcified in early stage of the healing process and gradually calcified ${ }^{17)}$. Our results clarified that the administration of MPMBP for 4 weeks induced the optimal maturation of newly formed bone. Thus, it was thought that MPMBP has no adverse effect on bone quality and induces the normal bone formation and maturation.

It is clinically important to rapidly fill the part of cortical bone defect in order to prevent connective tissue invasion and pathologic fractures. Bone strength depends on the quality, which is characterized by the geometry and shape of bones, turnover, mineral content, and collagen cross-links ${ }^{18)}$. Our study showed that the osseodensification of the cortical bone proceeded in both groups regardless of the administration of MPMBP and that the cortical bone significantly became dense early after discontinuing the administration of MPMBP. These findings allowed us to consider the timing of the cessation of MPMBP to prevent bone diseases.

Clinically, bisphosphonates are administered for the entire body and influence the bone of not only a target site but whole body, resulting in osteosclerosis. Sometimes bisphosphonates cause adverse reactions such as osteomyelitis and osteonecrosis ${ }^{12}$. We confirmed that the volume of newly formed bone decreased in the long term experiment. No excess bone formation or osteosclerosis was observed after withdrawal of MPMBP. These findings suggest that either discontinuing or shortening the administration period of MPMBP leads to appropriate bone remodeling. In clinical scene, zoledronic acid was intravenously administered in a lower dose of approximately $0.138 \mathrm{mM}^{18)}$ while in this study, MPMBP was locally administered in a high dose of $20 \mathrm{mM}$. Although a previous study indicated that the dose of $20 \mathrm{mM}$ was most effective to form new bone, appropriate dose and administration period should be reexamined for human.

In current clinical fields, BPs are used to improve the bones of whole body, while in this study, MPMBP was administered locally to effect minimum area. It is considered that the local administration of MPMBP influences the injection site instead of a wide range since micro-CT images demonstrated no significant difference of the length and breadth of the femoral bones between the experimental and control groups. Moreover, this study confirmed the significant increase of newly formed bone volume around the injection site in the superficial layers of the cortical bone and the part of the cortical bone defect in the bone marrow cavity. Thus, MPMBP seems to be effective in various clinical fields.
This study also proved that MPMBP caused no inflammation and promoted bone formation with appropriate bone remodeling. In other words, MPMBP would benefit the field of oral surgery because the local administration of MPMBP to the part requiring new bone formation can maintain new bone or bone bridge after invasive procedures such as tooth extraction, extirpation of a cyst and tumor, invasive reposition and fixation, and orthodontic surgery, with decreased risk of postoperative fracture. Furthermore, unlike bone substitute materials, the administration of MPMBP has a low risk of infection and no requirement of bone replacement surgery. Further investigation regarding dose and dosage and data collection are necessary to clinically use MPMBP in the future.

\section{Acknowledgements}

We thank Ms. Naoko Motoki and Ms. Miho Oikawa for excellent technical support.

\section{Conflict of Interest}

The authors have declared that no COI exists.

\section{References}

1. Michael JZ. Skeletal Healing. In: Primer on the metabolic bone diseases and disorders of mineral metabolism. 8th edition, ed by The American Society for Bone and Mineral Research, WILEY-BLACKWELL Inc., 90-98, 2013

2. Russow G, Jahn D, Appelt J, Märdian S, Tsitsilonis S and Keller J. Anabolic therapies in osteoporosis and bone regeneration. Int $\mathrm{J}$ Mol Sci 20(1): 83, 2019

3. Fleisch H. Development of bisphosphonates. Breast Cancer Res 4(1): 30-34, 2002

4. Fleisch H. Bisphosphonates-preclinical. In: Bisphosphonates in bone disease. Third edition, The Parthenon Publishing Group Inc., 32-57, 1997

5. Takaoka Y, Nagai H, Mori $H$ and Tanahashi M. The effect of TRK530 on experimental arthritis in mice. Biol Pharm Bull 20: 11471150, 1997

6. Baas J, Vestermark M, Jensen T, Bechtold J, Soballe K and Jakobsen T. Topical bisphosphonate augments fixation of bone-grafted hydroxyapatite coated implants, BMP-2 causes resorption-based decrease in bone. Bone 97: 76-82, 2017

7. Ortega AJ, Campbell PM, Hinton R, Naidu A and Buschang PH. Local application of zoledronate for maximum anchorage during space closure. Am J Orthod Dentofacial Orthop 142(6): 780-791, 2012

8. Lawson MA, Ebetino FH, Mazur A, Chantry AD, Paton-Hough J, Evans HR, Lath D, Tsoumpra MK, Lundy MW, Dobson RL, Quijano M, Kwaasi AA, Dunford JE, Duan X, Triffitt JT, Jeans G and Russell RGG. The pharmacological profile of a novel highly potent bisphosphonate, OX14 (1-Fluoro-2-(Imidazo-[1,2- $\alpha$ Pyridin-3-yl)-Ethyl-Bisphosphonate). J Bone Miner Res 32(9): 18601869,2017

9. Russell RG, Watts NB, Ebetino FH and Rogers MJ. Mechanisms of action of bisphosphonates: similarities and differences and their potential influence on clinical efficacy. Osteoporos Int 19(6): 733-759, 2008

10. Papapoulos SE. Bisphosphonate actions: physical chemistry revisited. Bone 38(5): 613-616, 2006

11. Takizawa A, Chiba M, Ota T, Yasuda M, Suzuki K, Kanemitsu T, Itoh T, Shinoda $\mathrm{H}$ and Igarashi $\mathrm{K}$. The novel bisphosphonate disodium dihydrogen-4-[(methylthio) phenylthio] methanebisphosphonate 
increases bone mass in post-ovariectomy rats. J Pharmacol Sci 131(1): 37-50, 2016

12. Prueter J and Dildeep A. Bisphosphonate related osteonecrosis of calvarial bone. Am J Otolaryngol 37(5): 470-472, 2016

13. Tanahashi M, Funaba Y, Tateishi A, Kawabe N and Nakadate-Matsushita T. TRK-530 inhibits accumulation of superoxide anions derived from human polymorphonuclear leukocytes and bone resorption induced by activated osteoclasts. Pharmacology 56: 125-130, 1998

14. Suzuki K, Takeyama S, Sakai Y, Yamada S and Shinoda H. Current topics in pharmacological research on bone metabolism: inhibitory effects of bisphosphonates on the differentiation and activity of osteoclasts. J Pharmacol Sci 100: 189-194, 2006

15. Shinoda H, Takeyama S, Suzuki K, Murakami S and Yamada S.
Pharmacological topics of bone metabolism: a nobel bisphosphonate for the treatment of periodontitis. J Pharmacol Sci 106: 555558,2008

16. Nishimura I, Shimizu Y and Ooya K. Effects of cortical bone perforation on experimental guided bone regeneration. Clin. Oral Implants Res 15: 293-300, 2004

17. Okata H, Nakamura M, Henmi A, Yamaguchi S, Mikami Y, Shimauchi $\mathrm{H}$ and Sasano Y. Calcification during bone healing in a standardized rat carvarial defect assessed by micro-CT and SEMEDX. Oral Dis 21: 74-82, 2015

18. Saito M, Shiraishi A, Ito M, Sakai S, Hayakawa N, Mihara M and Marumo K. Comparison of effects of alfacalcidol and alendronate on mechanical properties and bone collagen cross-links of callus in the fracture repair rat model. Bone, 46(4): 1170-1179, 2010 